Research Paper

\title{
Persistent Hepatic Inflammation Plays a Role in Hepatocellular Carcinoma After Sustained Virological Response in Patients with HCV Infection
}

Kazushige Nirei ${ }^{1}$, Tatsuo Kanda ${ }^{1}$, Hitomi Nakamura ${ }^{1}$, Shunichi Matsuoka ${ }^{1}$, Tadatoshi Takayama ${ }^{2}$, Masahiko Sugitani ${ }^{3}$ and Mitsuhiko Moriyama ${ }^{1}$

1. Division of Gastroenterology and Hepatology, Department of Internal Medicine

2. Department of Digestive Surgery and

3. Department of Pathology, Nihon University School of Medicine

$\triangle$ Corresponding author: Kazushige Nirei M.D., Ph.D., Division of Gastroenterology and Hepatology, Department of Internal Medicine, Nihon University School of Medicine, Japan, 30-1, Oyaguchikami-cho, Itabashi-ku, Tokyo 173-8610, Japan. Phone: +81-3-3972-8111 Ext.2424; Fax: +81-3-3956-8496; E-mail: nirei.kazushige@nihon-u.ac.jp

(c) Ivyspring International Publisher. This is an open access article distributed under the terms of the Creative Commons Attribution (CC BY-NC) license (https://creativecommons.org/licenses/by-nc/4.0/). See http://ivyspring.com/terms for full terms and conditions.

Received: 2017.10.03; Accepted: 2018.02.02; Published: 2018.03.08

\begin{abstract}
Objective: Hepatitis $\mathrm{C}$ virus (HCV) infection has long been treated with interferon therapy (IFN). Currently, more than $90 \%$ of IFN-treated patients show a sustained virological response (SVR) when also treated with ribavirin and/or a protease inhibitor. Histological inflammation and fibrosis improve in IFN-treated patients, which indicates HCV clearance. IFN also reduces the incidence of hepatocellular carcinoma (HCC). However, a small proportion of patients with SVR develop HCC. To investigate the causes of hepatic carcinogenesis after SVR, we compared the liver histological findings before IFN to those after the development of HCC.
\end{abstract}

Patients and methods: In total, 602 patients infected with type $C$ chronic hepatitis or with liver cirrhosis who received IFN therapy during the period from 1992 through 2015 were included in this study. We assessed 14 of the 287 patients who achieved an SVR.

Results: HCC was diagnosed by computed tomography, angiography or liver biopsy. The longest time from the SVR until HCC detection was 16.5 years, and the mean was $7.2 \pm 4.6$ years. Nine of the 14 patients underwent surgery and one radiofrequency ablation. The histological findings of 10 patients were available for comparison. The comparison of the histological findings before treatment with those after the HCC diagnosis revealed an amelioration of liver fibrosis and other inflammatory changes. All ten patients showed improvements in fibrosis and steatosis. However, we observed that mild inflammatory change persisted from 1.8 years to 16.5 years after the confirmation of SVR in all cases.

Conclusion: We suspect that persistent histological inflammation is one of the factors contributing to hepatocarcinogenesis (i.e., HCC development) even after successful treatment.

Key words: Chronic Hepatitis C; Sustained Virological Response; Hepatocellular Carcinoma; Histological Fibrosis; Persistent Histological Inflammation.

\section{Introduction}

The estimated prevalence of hepatitis $C$ virus ( $\mathrm{HCV}$ ) ranges from $2.3 \%$ to $2.8 \%$ worldwide [1]. $\mathrm{HCV}$ is a major cause of liver disease. In the United States and Japan, approximately $25 \%$ of infected patients develop hepatocellular carcinoma (HCC) and/or other forms of decompensated liver disease [2, 3]

Interferon (IFN) monotherapy has long been the mainstay of initial treatment for patients with chronic hepatitis C infection [4]. The next therapeutic advancement was ribavirin (RBV) combined with IFN 
or PEGylated IFN, a treatment regimen that achieved marked improvements in cure rates. In 2013, in an effort to increase the cure rate, the IFN plus RBV and a protease inhibitor (simeprevir) therapy was launched in Japan. Genotype $1 \mathrm{~b} \mathrm{HCV}$ patients achieved sustained virological response (SVR) rates exceeding $90 \%$ with this triple therapy regimen [5]. However, this therapeutic regimen was relatively ineffective in patients with a single IL28B nucleotide polymorphism [6]. Recently, direct acting antivirals (DAAs), exemplified by drugs such as Sofosbuvir and ledipasvir, reportedly have been achieving SVR rates of $95 \%$ to $100 \%$ [7-9]. In patients achieving SVR, improvements in liver function were demonstrated by reductions in aspartate transaminase (AST) and alanine transaminase (ALT) levels [10-12].

Histological improvement also has been documented in patients achieving SVR. Anti-viral therapies such as IFN contribute to the amelioration of hepatic inflammation and fibrosis [10-14].

On occasion, $\mathrm{HCV}$-infected patients showing an SVR in response to IFN develop HCC. According to one report, in patients greater than 65 years of age, there is no difference in the cumulative rates of hepatocarcinogenesis between those with an SVR and those who are non-responders to IFN [15]. After surgery for HCC, anti-viral therapy reportedly suppresses the recurrence of HCC [16]. These reports indicate that IFN therapy also suppresses the development of HCC. However, a small number of SVR patients develop HCC [17]. Our present study aimed to investigate the causes of carcinogenesis in chronic hepatitis $C$ patients with an SVR. We compared the histological findings before the administration of regimens including IFN to those at the time of the HCC diagnosis in patients with hepatitis C showing an SVR.

\section{Patients and methods}

\section{Study population}

A total of 602 patients with type C chronic hepatitis or liver cirrhosis who visited the Division of Gastroenterology and Hepatology at Nihon University Hospital to receive IFN therapy during the period from 1992 through 2015 were included in this study. For patients who were added to the study population from 2009 through 2012, combination therapy with Peg-IFN- $a 2 a$ or Peg-IFN- $a 2 b$ and RBV was administered for 6-12 months. From January 2015 to December 2015, combination therapy with Peg-IFN-a2a or Peg-IFN- $a 2 b$ and RBV was administered for 24 weeks, followed by simeprevir for 12 weeks. We previously reported the results that were obtained [18]. We considered an SVR to have been achieved in those patients who remained negative for serum HCV RNA for $>24$ weeks after the completion of IFN therapy (Figure 1).

At the time of starting the IFN administration, we measured the serum AST, ALT and $\gamma$-glutamyltransferase levels $(\mathrm{U} / \mathrm{L})$ and determined the serum platelet count $\left(\times 10^{4} / \mu \mathrm{L}\right)$ and $\mathrm{HCV}$ genotype. All patients gave informed consent to be included in this study, in accordance with the Declaration of Helsinki.

All patients were positive for the $\mathrm{HCV}$ antigen (by 2nd generation ELISA; Abbot, Tokyo, Japan) but negative for the serum hepatitis $B$ surface antigen (by HBsAg ELISA; Abbot), anti-nuclear antibody (by indirect immunofluorescence assay, IF; Special Reference Laboratory, Tokyo, Japan), anti-smooth muscle antibody (by IF), and anti-mitochondrial antibody (by IF).

We confirmed HCV positivity and measured the HCV RNA levels in the blood samples using the competitive reverse transcriptase-polymerase chain reaction and DNA probe methods (Special Reference Laboratory, Tokyo, Japan) and the Amplicor monitoring method (Amplicor HCV Monitor, Roche Diagnostic K.K., Tokyo, Japan) or Cobas TaqMan HCV method (Roche Diagnostics, Meylan, France).

Patients with Child B or Child C cirrhosis and those with a co-infection with the human immunodeficiency virus were excluded. Before the IFN treatment, the absence of malignant diseases was confirmed by blood biochemical, ultrasound, chest $\mathrm{X}$-ray and endoscopy examinations. Malignancy was an exclusion criterion.

\section{Liver Histology}

The liver tissues of the patients were intraoperatively harvested or obtained by needle biopsy. These tissue specimens were fixed in $2.5 \%$ formalin, embedded in paraffin, sectioned into 3-4 $\mu \mathrm{m}$ slices, and stained with hematoxylin and eosin (H\&E).

The fibrosis severity and activity scores were determined by the methods of Desmet [19], Knodell [9] and Ishak [20], as previously reported. Fibrosis was staged as F0 (no fibrosis), F1 (fibrous portal expansion), F2 (bridging fibrosis), F3 (bridging fibrosis with architectural distortion), and F4 (cirrhosis). The activity scores were graded as A0 (no activity), A1 (mild activity), A2 (moderate activity) and $\mathrm{A} 3$ (severe activity).

The scores of all biopsy specimens were independently assigned by the first author and one co-author (MM) without knowledge of the clinical characteristics of the patients.

The resected specimens were examined in detail. In all cases, the sites in the non-cancerous portion 
602 patients with type $\mathrm{C}$ chronic hepatitis or liver cirrhosis who received IFN therapy during the period from 1992 through 2015

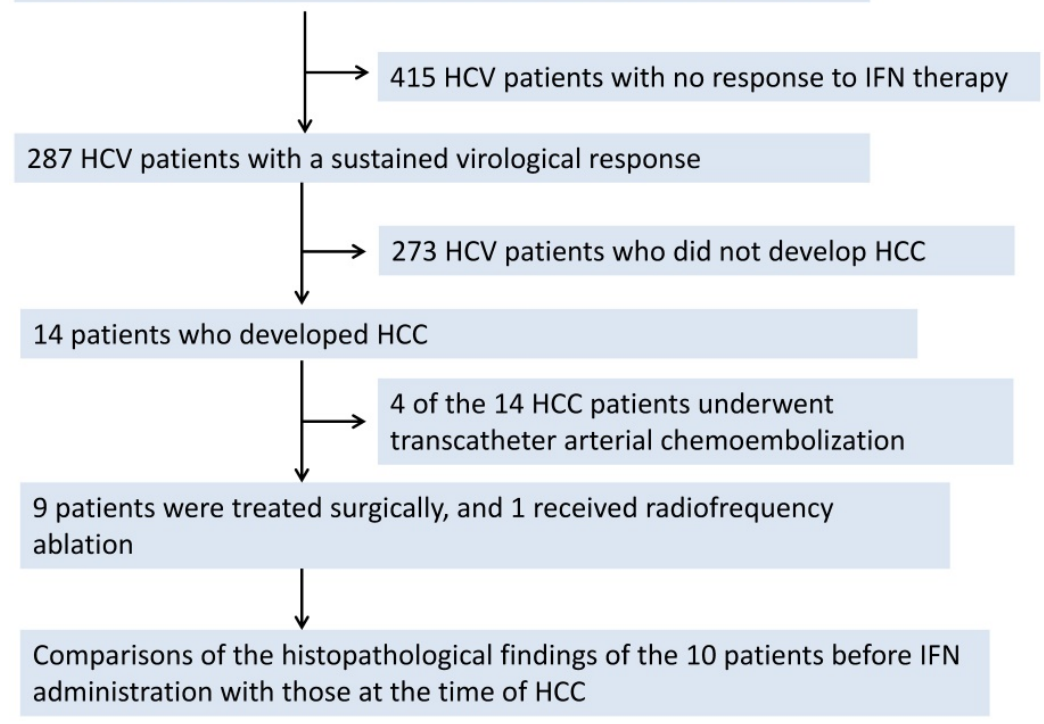

Figure 1. Flow chart

were examined at a distance of greater than $10 \mathrm{~mm}$ from the tumor.

\section{Statistical analysis}

Gender and genotype were compared using the $x^{2}$ test for independence. Liver histopathological findings and activity scores were compared using the Wilcoxon signed-ranks test. The remaining parameters are shown as the mean \pm SD and were compared using Student's t-test. These analyses were performed using JMP software (SAS Institute, Cary, NC). $\mathrm{P}$ values less than 0.05 were considered to indicate statistically significant differences.

\section{Results}

Figure 1 shows the flow chart for our subjects. Two hundred and eighty-seven patients (174 males and 113 females) achieved an SVR when treated with IFN. Fourteen of these patients developed HCC. Four of these $14 \mathrm{HCC}$ patients underwent transcatheter arterial chemoembolization. Nine patients were surgically treated, and one received radiofrequency ablation. The comparison of the histopathological findings before the IFN administration with those at the time of the HCC surgery for 10 patients was thus possible.

Nine of these 10 patients were male. The mean age of the patients at the time of the IFN administration was $61 \pm 6$ years, while that at the time of the HCC surgery was $67 \pm 6$ years.

\section{Laboratory data}

As shown in Table 1, we compared the laboratory data of the 10 patients before IFN with those at the time of the HCC diagnosis. The AST $(60 \pm 21 \mathrm{U} / \mathrm{L}$ vs. $23 \pm 24 \mathrm{U} / \mathrm{L}$, $\mathrm{p}<0.01)$ and ALT $(66 \pm 20 \mathrm{U} / \mathrm{L}$ vs. $22 \pm 12$ $\mathrm{U} / \mathrm{L}, \mathrm{p}<0.01$ ) levels were both reduced at the time of the HCC diagnosis compared to the pretreatment levels.

There were no significant changes in the alkaline phosphatase (ALP) level $(259 \pm 142 \mathrm{U} / \mathrm{L}$ vs. $242 \pm 60 \mathrm{U} / \mathrm{L}, \mathrm{p}=0.49)$, gamma-glutamyltransferase level $(55 \pm 23$ $\mathrm{U} / \mathrm{L}$ vs. $46 \pm 31 \mathrm{U} / \mathrm{L}, \mathrm{p}=0.47)$, total bilirubin level $(0.6 \pm 1.2 \mathrm{mg} / \mathrm{dL}$ vs. $0.7 \pm 0.4$ $\mathrm{mg} / \mathrm{dL}, \mathrm{p}=0.34)$, albumin level $(4.3 \pm 0.7$ g/dL vs. $4.2 \pm 0.3 \mathrm{~g} / \mathrm{dL}, \mathrm{p}=0.61)$, prothrombin time $(94 \pm 6 \%$ vs. $96 \pm 0.4 \%, p=0.33)$, platelet count $\left(15.9 \times 10^{4} / \mathrm{mm}^{3} \pm 5.0 \times 10^{4} /\right.$ $\mathrm{mm}^{3}$ vs. $17.6 \times 10^{4} / \mathrm{mm}^{3} \pm 3.8 \times 10^{4} / \mathrm{mm}^{3}$, $\mathrm{p}=0.30$ ) or alpha-fetoprotein (AFP) level $(7.9 \pm 3.4 \mathrm{ng} / \mathrm{mL}$ vs. $7.2 \pm 10.7 \mathrm{ng} / \mathrm{mL}$, $\mathrm{p}=0.12$ ). These data differed minimally between the time point of the SVR confirmation and when HCC was detected. Six patients had HCV genotype $1 b, 3$ had $\mathrm{HCV}$ genotype $2 \mathrm{a}$ and 1 had HCV genotype $2 b$. None of the 10 patients tested positive for Hepatitis B virus (HBV) DNA by the TaqMan polymerase chain reaction. Four of the patients were positive for $\mathrm{HB}$ surface antibodies (HBsAb), and 5 were positive for $\mathrm{HB}$ core antibodies ( $\mathrm{HBcAb})$.

Table 1. Comparisons of laboratory data collected from 10 patients before IFN treatment and after HCC resection.

\begin{tabular}{llll}
\hline & Liver biopsy & HCC diagnosis & P \\
\hline Gender F:M (n:n) & $1: 9$ & $1: 9$ & \\
Age (years) & $61 \pm 6$ & $67 \pm 6$ & \\
AST (U/L) & $60 \pm 21$ & $23 \pm 4$ & 0.01 \\
ALT (U/L) & $66 \pm 20$ & $22 \pm 12$ & 0.01 \\
Y-Glutamyltransferase (U/L) & $55 \pm 23$ & $46 \pm 31$ & 0.47 \\
Total bilirubin (mg/dL) & $0.6 \pm 1.2$ & $0.7 \pm 0.4$ & 0.34 \\
ALP (U/L) & $259 \pm 142$ & $242 \pm 60$ & 0.49 \\
Albumin (g/dL) & $4.3 \pm 0.7$ & $4.2 \pm 0.3$ & 0.61 \\
Prothrombin time (\%) & $94 \pm 6$ & $96 \pm 4$ & 0.33 \\
Platelets (×104/mm 3 ) & $15.9 \pm 5.0$ & $17.6 \pm 3.8$ & 0.3 \\
AFP (ng/mL) & $7.9 \pm 3.4$ & $7.2 \pm 10.7$ & 0.12 \\
Serotype 1:2 & $6: 4$ & & \\
HBcAb (+:-) & $5: 5$ & & \\
HBsAb (+:-) & $4: 6$ & & \\
HBV DNA & not detected & not detected & \\
\hline
\end{tabular}

\section{Fib-4 index}

To compare the histological scores, we checked the Fib-4 index (Table 2). However, there were no significant changes $(\mathrm{p}=0.96)$.

\section{Histopathological findings}

Figure 2 ( $a, b, c$, and d) shows the carcinogenic 
changes documented approximately 3.7 years after the confirmation of an SVR in an IFN-treated patient (Table 3; Patient No. 4). Figure 2a shows an H\&E-stained specimen at $\times 4$ magnification, while Figure $2 b$ shows an H\&E-stained specimen at $\times 20$ magnification. Both of these images were obtained prior to IFN administration. Figure 2c shows an H\&E-stained specimen at $\times 4$ magnification, while Figure $2 \mathrm{~d}$ shows an H\&E-stained specimen at $\times 20$ magnification. Both of these images were obtained at the time of HCC resection. The fibrosis score shows a slight improvement. However, necrotic patches, as well as steatosis, can still be seen.

Table 2. A comparison of the histological scores in regards to the Fib-4 index. There were no significant changes observed $(p=0.96)$.

\begin{tabular}{|c|c|c|}
\hline \multicolumn{2}{|c|}{ Fib4 index } & \multirow[b]{2}{*}{ At HCC diagnosis } \\
\hline Patient No. & Before IFN & \\
\hline 1 & 3.98 & 1.95 \\
\hline 2 & 3.18 & 2.00 \\
\hline 3 & 1.46 & 1.98 \\
\hline 4 & 3.45 & 1.38 \\
\hline 5 & 3.54 & 1.76 \\
\hline 6 & 1.63 & 1.07 \\
\hline 7 & 1.94 & 2.13 \\
\hline 8 & 3.94 & 2.12 \\
\hline 9 & 3.32 & 1.74 \\
\hline 10 & 1.72 & $\mathrm{P}=0.96$ \\
\hline
\end{tabular}
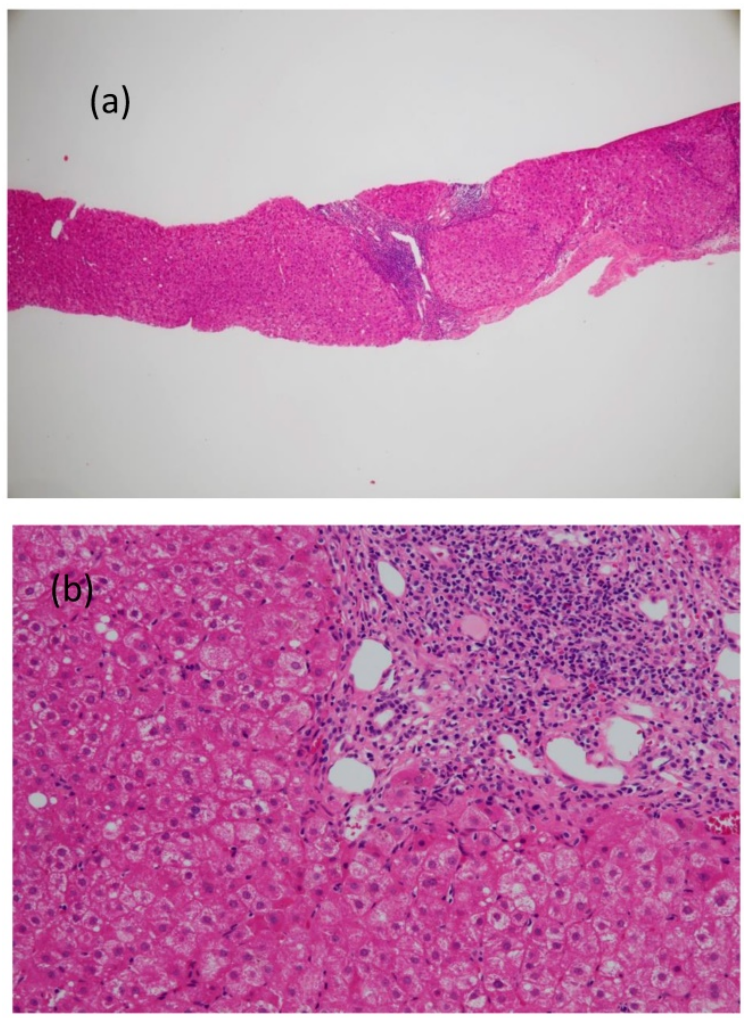

Figure $3(a, b, c$, and d) shows the carcinogenic changes documented approximately 8.5 years after the confirmation of an SVR in an IFN-treated patient (Table 3; Patient No. 8).

Figure 3 a shows an H\&E-stained specimen at $\times 4$ magnification, while Figure $3 \mathrm{~b}$ shows an H\&E-stained specimen at $\times 20$ magnification. Both of these images were obtained prior to IFN administration. Figure $3 \mathrm{c}$ shows an H\&E-stained specimen at $\times 4$ magnification, while Figure $4 \mathrm{~d}$ shows an H\&E-stained specimen at $\times 20$ magnification. Both of these images were obtained during HCC resection. The fibrosis score showed an improvement from F4 to F3, and slight bridging necrosis was detected. Steatosis, as well as inflammation of the portal area, was still present.

Figure 4 (a, b, c, and d) shows the carcinogenic changes documented approximately 16.5 years after the confirmation of an SVR in an IFN-treated patient (Table 3; Patient No. 10). Figure 4a shows an H\&E-stained specimen at $\times 4$ magnification, while Figure $4 \mathrm{~b}$ shows an H\&E-stained specimen at $\times 20$ magnification. Both of these images were obtained before IFN administration. Figure $4 \mathrm{c}$ shows an H\&E-stained specimen at $\times 4$ magnification, while Figure $4 \mathrm{~d}$ shows an H\&E-stained specimen at $\times 20$
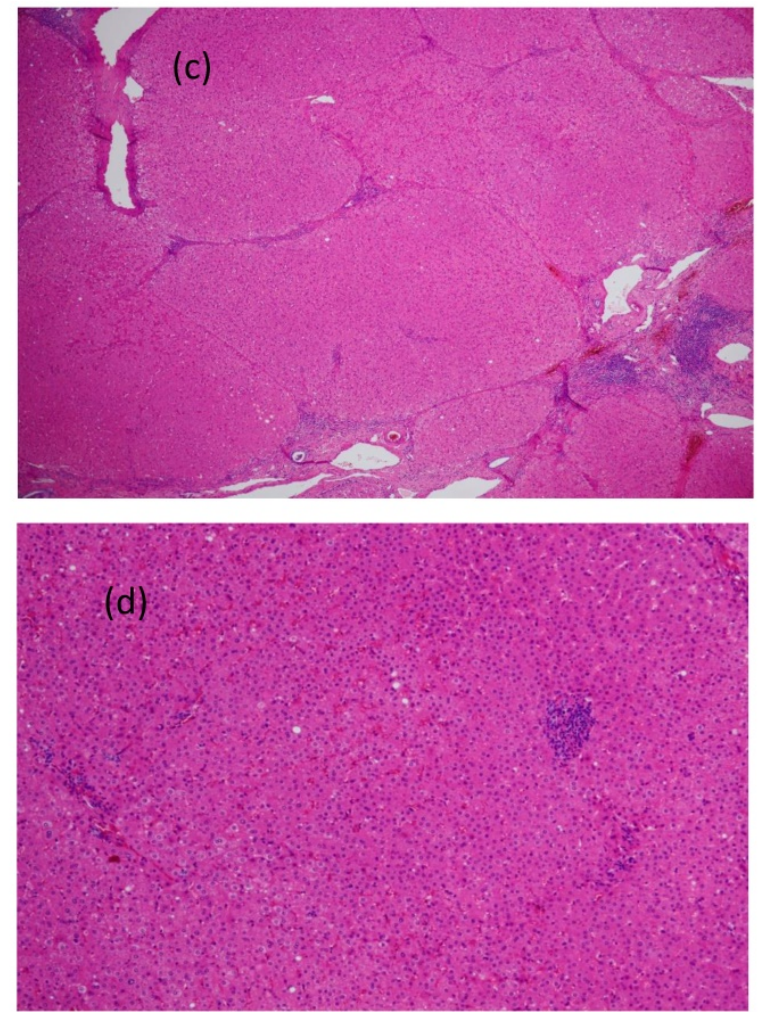

Figure 2. The carcinogenic changes documented approximately 3.7 years after the confirmation of an SVR in an IFN-treated patient are shown. Figure $2 \mathrm{a}$ shows an $\mathrm{H} \& \mathrm{E}$-stained specimen at $\times 4$ magnification, while Figure $2 \mathrm{~b}$ shows an H\&E-stained specimen at $\times 20$ magnification. Both images were obtained prior to IFN administration. Figure $2 c$ shows an H\&E-stained specimen at $\times 4$ magnification, while Figure $2 d$ shows an H\&E-stained specimen at $\times 20$ magnification. Both images were obtained at the time of HCC resection. The fibrosis score shows a slight improvement. However, necrotic patches, as well as steatosis, can still be seen. 
magnification. Both of these were obtained during the surgery for HCC. The specimen that was obtained 16 years earlier clearly shows fibrosis. At the time of HCC resection, the fibrosis had improved from F3 to F1. Inflammation of the portal area was also reduced, though necrotic patches remained (Figure 4d). The inflammation within the parenchyma and necrosis showed improvement, but there were still areas that were affected. No lymphoid follicle formation was detected in the portal area.

\section{Hepatic fibrosis grade and histopathological activity score}

Table 3 shows the fibrosis grades of the patients before IFN treatment. One patient had stage F1, 2 had stage F2, 4 had stage F3, and 3 had stage F4. At the time of the HCC resection, 6 patients had stage F1, 1 had stage F2, 3 had stage F3, and none had stage F4. There were statistically significant differences between the fibrosis stages before IFN and at the time of surgery for HCC $(\mathrm{P}=0.01)$. None of the patients showed a worsening of fibrosis.

Table 3. Comparisons of fibrosis grades and inflammation activity scores before IFN treatment and after HCC resection.

\begin{tabular}{|c|c|c|c|c|c|c|c|c|}
\hline Patient No. & $\begin{array}{l}\text { Time from } \\
\text { IFN to HCC } \\
\text { (y) }\end{array}$ & $\begin{array}{l}\text { Age at IFN } \\
\text { (y) }\end{array}$ & Gender & Fibrosis & Activity & Fibrosis & Activity & IFN therapy \\
\hline 1 & 1.8 & 62.4 & $\mathrm{M}$ & 2 & 2 & 1 & 1 & Monotherapy \\
\hline 2 & 3.5 & 67.9 & M & 3 & 2 & 2 & 2 & Peg IFN a2a+ ribavirin \\
\hline 3 & 3.5 & 63.7 & M & 1 & 2 & 1 & 1 & Monotherapy \\
\hline 4 & 3.7 & 51.9 & M & 4 & 2 & 3 & 2 & Peg IFN a $2 a+$ ribavirin \\
\hline 5 & 6.6 & 58.3 & M & 4 & 3 & 1 & 1 & Peg IFN a2a+ ribavirin \\
\hline 6 & 7 & 58.8 & $\mathrm{~F}$ & 2 & 2 & 1 & 2 & Monotherapy \\
\hline 7 & 7.8 & 67.6 & M & 3 & 1 & 3 & 1 & Monotherapy \\
\hline 8 & 8.5 & 57.2 & M & 4 & 3 & 3 & 2 & Monotherapy \\
\hline 9 & 13.3 & 46.7 & M & 3 & 2 & 1 & 1 & Monotherapy \\
\hline 10 & 16.5 & 61.3 & $\mathrm{M}$ & 3 & 3 & 1 & 1 & Monotherapy \\
\hline
\end{tabular}

The differences were statistically significant $(\mathrm{p}=0.023)$. None of the patients showed an increase in either fibrosis grade nor activity score.
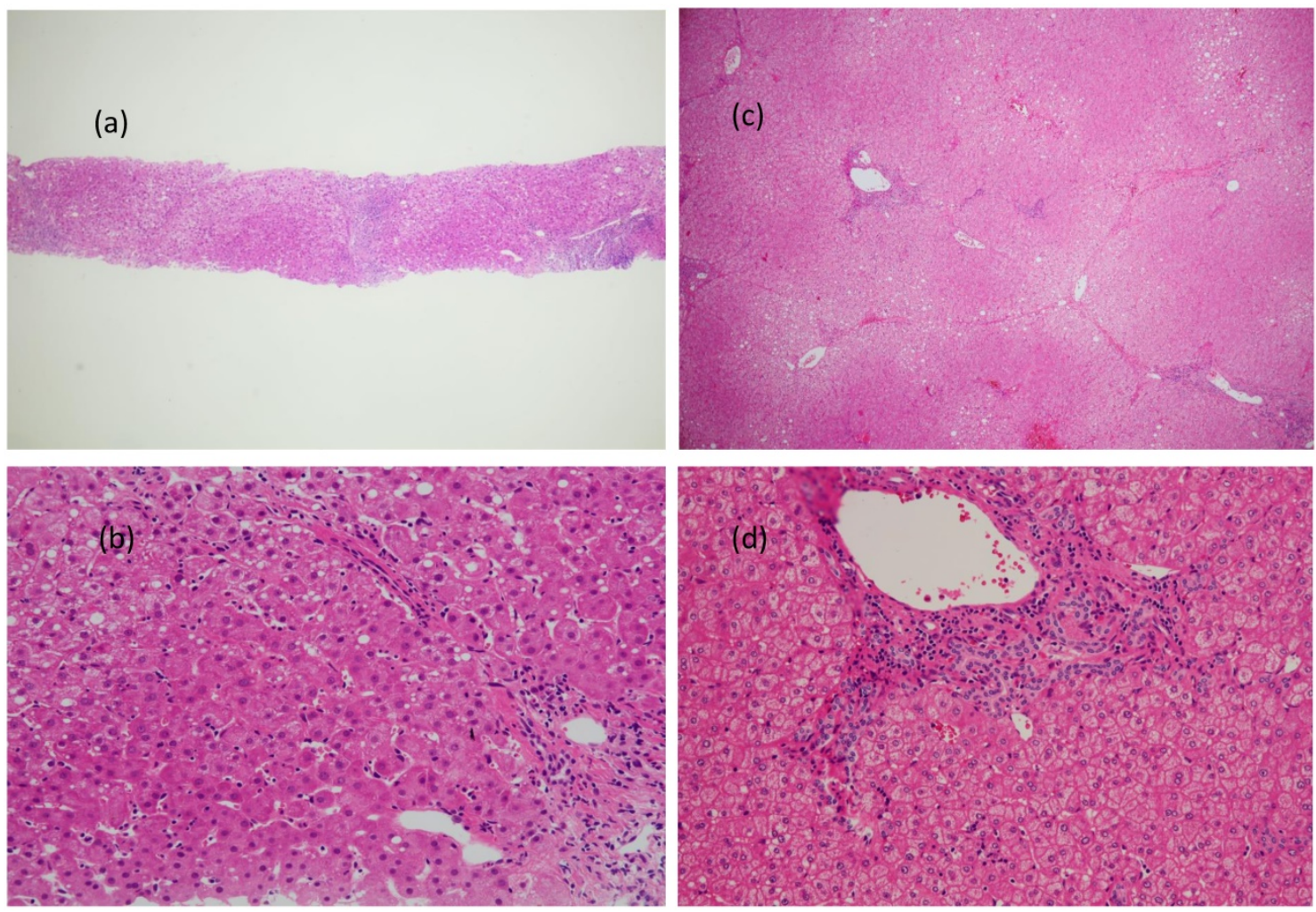

Figure 3. Carcinogenic changes documented approximately 8.5 years after the confirmation of an SVR in an IFN-treated patient (corresponding to Patient No. 8; Table 3) are shown. Figure 3a shows an H\&E-stained specimen at $\times 4$ magnification, while Figure $3 \mathrm{~b}$ shows an $\mathrm{H} \& \mathrm{E}-\mathrm{stained}$ specimen at $\times 20$ magnification. Both images were obtained prior to IFN administration. Figure $3 \mathrm{c}$ shows an $\mathrm{H} \& \mathrm{E}$-stained specimen at $\times 4$ magnification, while Figure $3 \mathrm{~d}$ shows an $\mathrm{H} \& \mathrm{E}-\mathrm{stained}$ specimen at $\times 20$ magnification. Both images were obtained during HCC resection. The fibrosis score showed improvement from F4 to F3, and slight bridging necrosis was detected. Steatosis also showed marked improvement, but inflammation persisted in the portal area. 

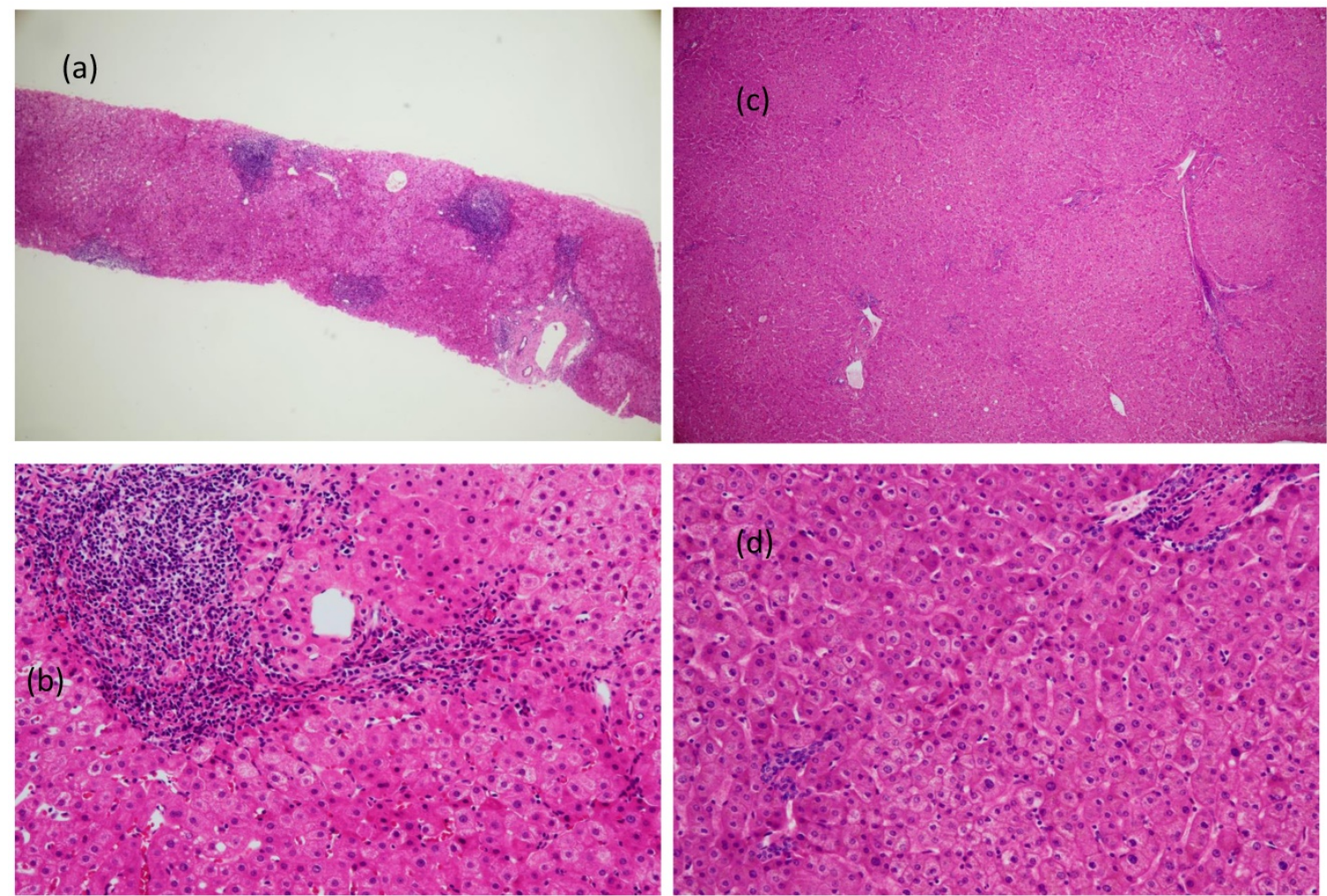

Figure 4. Carcinogenic changes documented approximately 16.5 years after the confirmation of an SVR in an IFN-treated patient are shown. Figure 4a shows an $\mathrm{H} \& \mathrm{E}$-stained specimen at $\times 4$ magnification, while Figure $4 \mathrm{~b}$ an H\&E-stained specimen at $\times 20$ magnification. Both images were obtained before IFN administration. Figure $4 \mathrm{c}$ shows an H\&E-stained specimen at $\times 4$ magnification, while Figure $4 \mathrm{~d}$ shows an H\&E-stained specimen at $\times 20$ magnification. Both images were obtained during the surgery for HCC. The specimen that was obtained 16 years earlier clearly shows fibrosis. At the time of HCC resection, the fibrosis grade had improved from F3 to F1. The inflammation of the portal area was also reduced, though necrotic patches can still be seen in the specimen (Figure 4d). The inflammation within the parenchyma and necrosis showed improvement, but there were still areas that were affected. No lymphoid follicle formation was detected in the portal area.

As for the activity scores before the IFN therapy, 1 patient had grade A1, 6 had grade A2, and 3 had grade A3. At the time of the HCC resection, 6 patients had grade A1 and 4 had grade A2. There were statistically significant differences between the activity scores before IFN and at the time of surgery for HCC $(\mathrm{P}=0.023)$. None of the patients showed a worsening of inflammation. However, all the patients had histological findings indicative of inflammation.

\section{Steatosis of the liver}

Nine of the 10 patients had steatosis before IFN administration. Even after being treated with IFN, either alone or in combination with other agents, all 9 patients still had steatosis.

\section{Discussion}

Histological specimens that allow comparisons to be made between the findings prior to IFN treatment and those at the time of HCC resection are not widely available. One of the patients in the present study, who developed HCC with an SVR more than 16 years after the administration of IFN, is particularly rare and may provide insights into the long-term process of hepatic carcinogenesis.
In our study, the shortest time between the IFN administration and HCC detection was 1.8 years. This patient showed no evidence of HCC on either computed tomography or ultrasonography prior to IFN treatment. While it is not possible to confirm that none of these 10 patients had HCC at the time of the SVR, the longest period from the SVR until HCC detection was 16.5 years, making carcinoma at the time of SVR confirmation extremely unlikely. Before starting this study, it was our view that histological inflammation would disappear completely after the attainment of an SVR. However, we observed that a mild inflammatory change persisted from 1.8 years to 16.5 years after the confirmation of an SVR in some cases.

To our knowledge, there are no reports indicating that the HCV RNA genome becomes integrated into the human genome. $\mathrm{HCV}$ is apparently not carcinogenic on its own. We thus speculate that a prolonged histological inflammation is one of the causes of carcinogenesis and that it can be effective even after HCV has been eradicated.

According to another report, very low levels of $\mathrm{HCV}$ may persist in hepatocytes. Maylin et al. reported that serum HCV RNA was negative but 
HCV RNA was detected in liver biopsy specimens from 2 of their 114 hepatitis C patients (1.7\%) [21]. The hepatocytes of our patients were not tested for HCV RNA, and we thus cannot rule out the possibility of there being low levels of the virus persisting in liver cells.

The histological fibrosis of the patient diagnosed with HCC 1.8 years after attaining a SVR improved from F2 to F1. The patients who had liver cirrhosis 3.7 years and 6.6 years after IFN treatment showed improvements from $\mathrm{F} 4$ to $\mathrm{F} 2$ and from $\mathrm{F} 4$ to $\mathrm{F} 1$, respectively. In our patient with the longest period between IFN administration and HCC, which was 16.5 years, fibrosis had remarkably improved from $\mathrm{F} 3$ to F1. Thus, all of the patients showed an amelioration but not complete disappearance of fibrosis.

Comparisons of the histological features of patients with chronic hepatitis $\mathrm{C}$ before and after IFN treatment have been reported. Biopsies that were obtained after an SVR demonstrated that $57 \%-94 \%$ of hepatitis C patients with an SVR showed reductions of inflammation and fibrosis [10, 11, 13, 22]. However, according to some reports, $1 \%-14 \%$ of patients showed no changes in the histological findings [20, 22]. In this study, despite them both showing improvements, inflammation and fibrosis were still detectable in all patients.

There are many reports describing diabetes and fatty liver as being involved in carcinogenesis $[12,13$, $23,24]$. In all but one of our patients, steatosis also persisted after the confirmation of an SVR. Previously, we reported that steatosis influences the clinical profiles and long-term outcomes of IFN-treated chronic hepatitis $\mathrm{C}$ and liver cirrhosis patients. In our present report, 12 of the 14 patients had steatosis of their liver tissues before IFN administration. 'Steatosis', 'lobular inflammation', 'hepatocyte ballooning', 'Mallory-Denk bodies', and 'fibrosis' were documented in our patients. However, at the time of the HCC resection, these histological findings were relatively mild. We thus suggest that steatosis is not a cause of residual and/or ongoing inflammation.

Primary biliary cirrhosis and autoimmune hepatitis have also been suggested as being causes of inflammation in hepatitis $C$ patients. However, in this study, all of the patients were negative for serum ANA and AMA M2 [anti-nuclear and anti-mitochondrial antibodies], making primary biliary cirrhosis and autoimmune hepatitis unlikely. However, neither can be definitively ruled out.

We previously reported on occult HBV infection in HCV patients [25]. According to one report, occult HBV infection is not associated with disease progression in patients with chronic $\mathrm{HCV}$ infection [26]. HBV co-infection reportedly can also cause HCC.
Of the previously reported HCC patients, there is a greater number of those who were positive for $\mathrm{HBsAb}$ and $\mathrm{HBcAb}$ than those who were negative for $\mathrm{HBsAb}$ and $\mathrm{HBcAb}$. HCV patients who were $\mathrm{HBcAb}$ positive developed HCC at a higher rate than those who were $\mathrm{HBcAb}$ negative. Even among those $\mathrm{HCV}$ patients who were $\mathrm{HBsAb}$ negative, being $\mathrm{HBcAb}$ positive was reported as being a poor prognostic factor $[25,27$, 28]. In this study, all of the included patients were negative for serum HBs antigen and HBV DNA, which was evidenced by the TaqMan polymerase chain reaction. Six of the 10 patients were positive for $\mathrm{HBCAb}$. These 6 patients had occult HBV infection but HBV activity that was not high. The other 4 patients were negative for $\mathrm{HBV}$ markers and thus did not have occult HBV infection. HBV would therefore be an unlikely source of the histological inflammation in our patients.

Of the 10 patients in our study, none were heavy alcohol drinkers, and there seems to be no relationship between alcohol drinking and the histological findings.

Compared to the pretreatment levels, the AST and ALT levels after the HCC diagnosis were both reduced $(\mathrm{P}<0.01)$, while the glutamic-pyruvic transaminase level, total bilirubin level, ALP level, albumin level, platelet counts and prothrombin time were essentially unchanged. All of the patients had normal AFP values before starting IFN treatment. After HCC detection, only one patient was positive for AFP (37.5 ng/dL). Therefore, it was difficult to predict HCC solely based on the blood test findings.

The platelet count after an SVR, which was measured during the HCC treatment, was not improved $(p=0.33)$ from that before IFN. Furthermore, the Fib-4 index showed no statistically significant difference after an SVR compared to before IFN $(p=0.96)$ (Table 2). We suggest that HCC that develops after an SVR is not able to be predicted by the patient's laboratory data.

Makiyama et al. reported that of $3262 \mathrm{HCV}$ patients treated with IFN, 1197 (37\%) attained an SVR. During follow-up, 27 of these patients (2.3\%) developed HCC. The cumulative HCC incidence was unrelated to both the serum transaminase levels and histological staging [17]. Our findings also suggest that HCC that develops after an SVR is not able to be predicted by the patient's laboratory data. In another study that focuses on the prognosis of patients who develop HCC after an SVR, $8147 \mathrm{HCV}$ patients treated with IFN were followed up for 6 years. The HCC occurrence rate was $0.64 \%$ /year in the HCV patient population. Patients who had liver cirrhosis and those who were older than 50 years were at a high risk of carcinogenesis [29]. All of the HCC patients in our 
study were also older than 50 years. The prior report and our present data suggest that patients with liver cirrhosis and those older than 50 years should receive a meticulous long-term follow-up. Furthermore, the inflammatory histological findings did not correspond to abnormalities of the laboratory data.

Ikeda reported on $7715 \mathrm{HCV}$ patients who showed an eradication of $\mathrm{HCV}$ in response to IFN. Thirty-eight of these patients developed HCC later on. The follow-up for the patients was nearly 8 years. Compared to those without HCC, the patients who developed HCC showed high histological activity in the biopsy specimens [30]. In our present study, the patients who achieved an SVR did not necessarily show high histological activity prior to IFN treatment.

Recently, DAAs such as Sofosbuvir and ledipasvir have become mainstays of treatment. These drugs have achieved SVR rates exceeding 95\% [7-9]. Toyoda et al. reported that patients who received DAA drugs after the confirmation of an SVR were at a higher risk for HCC than those who were given IFN. Their report suggests that IFN-based and IFN-free therapies should be compared in terms of hepatocarcinogenesis suppression along with HCV eradication [31]. As for HCV treatment with DAAs, the development of HCC is reportedly suppressed in patients with an SVR who have been treated with DAAs [32]. However, in other reports, the development of HCC did not appear to be suppressed in those receiving treatment with DAAs $[33,34]$.

This issue merits further study. Histological examination of the non-cancerous areas around an HCC after an SVR obtained with direct-acting antiviral drugs might be very useful for an optimal treatment selection. We plan to have a long-term follow-up of the patients treated with DAAs in our study. If such patients develop HCC, their histological findings before treatment should be compared with those of the resected HCC specimen.

\section{Acknowledgements}

The authors would like to thank all of the patients and the research and clinic staff members of the Liver Unit of the Nihon University School of Medicine.

The study protocol number of our institute is RK 10091015.

\section{Conflicts of interest}

The authors have no conflicts of interest to disclose.

\section{References}

1. Mohd Hanafiah K, Groeger J, Flaxman AD, Wiersma ST. Global epidemiology of hepatitis $C$ virus infection: new estimates of age-specific antibody to HCV seroprevalence. Hepatology (Baltimore, Md). 2013; 57: 1333-42.

2. Chak E, Talal AH, Sherman KE, Schiff ER, Saab S. Hepatitis C virus infection in USA: an estimate of true prevalence. Liver international : official journal of the International Association for the Study of the Liver. 2011; 31: 1090-101.

3. Freeman AJ, Dore GJ, Law MG, Thorpe M, Von Overbeck J, Lloyd AR, et al. Estimating progression to cirrhosis in chronic hepatitis $\mathrm{C}$ virus infection. Hepatology (Baltimore, Md). 2001; 34: 809-16.

4. McHutchison JG, Gordon SC, Schiff ER, Shiffman ML, Lee WM, Rustgi VK, et al. Interferon alfa-2b alone or in combination with ribavirin as initial treatment for chronic hepatitis C. Hepatitis Interventional Therapy Group. The New England journal of medicine. 1998; 339: 1485-92.

5. Manns M, Marcellin P, Poordad F, de Araujo ES, Buti M, Horsmans Y, et al. Simeprevir with pegylated interferon alfa $2 \mathrm{a}$ or $2 \mathrm{~b}$ plus ribavirin in treatment-naive patients with chronic hepatitis $C$ virus genotype 1 infection (QUEST-2): a randomised, double-blind, placebo-controlled phase 3 trial. Lancet (London, England). 2014; 384: 414-26.

6. Tanaka Y, Nishida N, Sugiyama M, Kurosaki M, Matsuura K, Sakamoto N, et al. Genome-wide association of IL28B with response to pegylated interferon-alpha and ribavirin therapy for chronic hepatitis C. Nature genetics. 2009; 41: 1105-9.

7. Afdhal N, Reddy KR, Nelson DR, Lawitz E, Gordon SC, Schiff E, et al. Ledipasvir and sofosbuvir for previously treated HCV genotype 1 infection. The New England journal of medicine. 2014; 370: 1483-93.

8. Afdhal N, Zeuzem S, Kwo P, Chojkier M, Gitlin N, Puoti M, et al. Ledipasvir and sofosbuvir for untreated HCV genotype 1 infection. The New England journal of medicine. 2014; 370: 1889-98

9. Kowdley KV, Gordon SC, Reddy KR, Rossaro L, Bernstein DE, Lawitz E, et al. Ledipasvir and sofosbuvir for 8 or 12 weeks for chronic HCV without cirrhosis. The New England journal of medicine. 2014; 370: 1879-88.

10. Poynard T, Moussalli J, Munteanu M, Thabut D, Lebray P, Rudler M, et al. Slow regression of liver fibrosis presumed by repeated biomarkers after virological cure in patients with chronic hepatitis C. Journal of hepatology. 2013; 59: 675-83.

11. Toccaceli F, Laghi V, Capurso L, Koch M, Sereno S, Scuderi M. Long-term liver histology improvement in patients with chronic hepatitis $C$ and sustained response to interferon. Journal of viral hepatitis. 2003; 10: 126-33.

12. Moriyama $M$, Matsumura $H$, Nirei $K$, Arakawa $Y$, Yamagami H, Ogawa $M$, et al. Factors influencing treatment efficacy of 24-week combination therapy with interferon alpha- $2 b$ plus ribavirin for chronic hepatitis $C$. Digestive diseases and sciences. 2007; 52: 2418-26.

13. Poynard T, McHutchison J, Manns M, Trepo C, Lindsay K, Goodman Z, et al. Impact of pegylated interferon alfa- $2 \mathrm{~b}$ and ribavirin on liver fibrosis in patients with chronic hepatitis C. Gastroenterology. 2002; 122: 1303-13.

14. Shiratori Y, Imazeki F, Moriyama M, Yano M, Arakawa Y, Yokosuka O, et al. Histologic improvement of fibrosis in patients with hepatitis $C$ who have sustained response to interferon therapy. Annals of internal medicine. 2000; 132: 517-24.

15. Kobayashi S, Takeda T, Enomoto M, Tamori A, Kawada N, Habu D, et al. Development of hepatocellular carcinoma in patients with chronic hepatitis C who had a sustained virological response to interferon therapy: a multicenter, retrospective cohort study of 1124 patients. Liver international : official journal of the International Association for the Study of the Liver. 2007; 27: 186-91.

16. Hsu YC, Ho HJ, Wu MS, Lin JT, Wu CY. Postoperative peg-interferon plus ribavirin is associated with reduced recurrence of hepatitis $C$ virus-related hepatocellular carcinoma. Hepatology (Baltimore, Md). 2013; 58: 150-7.

17. Makiyama A, Itoh $\mathrm{Y}$, Kasahara A, Imai $\mathrm{Y}$, Kawata S, Yoshioka K, et al. Characteristics of patients with chronic hepatitis C who develop hepatocellular carcinoma after a sustained response to interferon therapy. Cancer. 2004; 101: 1616-22.

18. Nirei $\mathrm{K}$, Matsumura $\mathrm{H}$, Kumakawa $\mathrm{M}$, Matsumoto $\mathrm{N}$, Nakamura $\mathrm{H}$, Yamagami $\mathrm{H}$, et al. Steatosis influences the clinical profiles and long-term outcomes of interferon-treated chronic hepatitis $C$ and liver cirrhosis patients. International journal of medical sciences. 2017; 14: 45-52.

19. Desmet VI, Gerber M, Hoofnagle JH, Manns M, Scheuer PJ. Classification of chronic hepatitis: diagnosis, grading and staging. Hepatology (Baltimore, Md). 1994; 19: 1513-20.

20. Ishak K, Baptista A, Bianchi L, Callea F, De Groote J Gudat F, et al. Histological grading and staging of chronic hepatitis. Journal of hepatology. 1995; 22: 696-9.

21. Maylin S, Martinot-Peignoux M, Moucari R, Boyer N, Ripault MP, Cazals-Hatem D, et al. Eradication of hepatitis $C$ virus in patients successfully treated for chronic hepatitis C. Gastroenterology. 2008; 135: 821-9.

22. Marcellin P, Boyer N, Gervais A, Martinot M, Pouteau M, Castelnau C, et al. Long-term histologic improvement and loss of detectable intrahepatic HCV RNA in patients with chronic hepatitis $C$ and sustained response to interferon-alpha therapy. Annals of internal medicine. 1997; 127: 875-81.

23. Kawamura Y, Arase Y, Ikeda K, Seko Y, Imai N, Hosaka T, et al. Large-scale long-term follow-up study of Japanese patients with non-alcoholic Fatty liver disease for the onset of hepatocellular carcinoma. The American journal of gastroenterology. 2012; 107: 253-61. 
24. Kurosaki M, Hosokawa T, Matsunaga K, Hirayama I, Tanaka T, Sato M, et al. Hepatic steatosis in chronic hepatitis $C$ is a significant risk factor for developing hepatocellular carcinoma independent of age, sex, obesity, fibrosis stage and response to interferon therapy. Hepatology research : the official journal of the Japan Society of Hepatology. 2010; 40: 870-7.

25. Nirei K, Kaneko M, Moriyama M, Arakawa Y. The clinical features of chronic hepatitis $C$ are not affected by the coexistence of hepatitis B virus DNA in patients negative for hepatitis B surface antigen. Intervirology. 2000; 43: 95-101.

26. Cho J, Lee SS, Choi YS, Jeon Y, Chung JW, Baeg JY, et al. Occult hepatitis B virus infection is not associated with disease progression of chronic hepatitis $\mathrm{C}$ virus infection. World journal of gastroenterology. 2016; 22: 9427-36.

27. Nan X, Shi S, Yu C, Zhuang H. Meta-analysis of the association between anti-HBc seropositivity and a poor prognosis of chronic HCV infection. Hepatology research : the official journal of the Japan Society of Hepatology. 2010; 40: 1176-87.

28. Matsuoka S, Nirei K, Tamura A, Nakamura H, Matsumura H, Oshiro S, et al. Influence of occult hepatitis $\mathrm{B}$ virus coinfection on the incidence of fibrosis and hepatocellular carcinoma in chronic hepatitis C. Intervirology. 2008; 51: 352-61.

29. Janjua NZ, Chong M, Kuo M, Woods R, Wong J, Yoshida EM, et al. Long-term effect of sustained virological response on hepatocellular carcinoma in patients with hepatitis C in Canada. Journal of hepatology. 2017; 66: 504-13.

30. Ikeda M, Fujiyama S, Tanaka M, Sata M, Ide T, Yatsuhashi H, et al. Clinical features of hepatocellular carcinoma that occur after sustained virological response to interferon for chronic hepatitis C. Journal of gastroenterology and hepatology. 2006; 21: 122-8.

31. Toyoda H, Tada T, Takaguchi K, Senoh T, Shimada N, Hiraoka A, et al. Differences in background characteristics of patients with chronic hepatitis C who achieved sustained virologic response with interferon-free versus interferon-based therapy and the risk of developing hepatocellular carcinoma after eradication of hepatitis $C$ virus in Japan. Journal of viral hepatitis. 2016.

32. Kanwal F, Kramer J, Asch SM, Chayanupatkul M, Cao Y, El-Serag HB. Risk of Hepatocellular Cancer in HCV Patients Treated With Direct Acting Antiviral Agents. Gastroenterology. 2017.

33. Conti F, Buonfiglioli F, Scuteri A, Crespi C, Bolondi L, Caraceni P, et al. Early occurrence and recurrence of hepatocellular carcinoma in HCV-related cirrhosis treated with direct-acting antivirals. Journal of hepatology. 2016; 65: 727-33.

34. Reig M, Marino Z, Perello C, Inarrairaegui M, Ribeiro A, Lens S, et al. Unexpected high rate of early tumor recurrence in patients with HCV-related HCC undergoing interferon-free therapy. Journal of hepatology. 2016; 65: 719-26. 